\title{
Robust adaptive algorithm for active control of impulsive noise
}

\author{
Alina Mirza*, Ayesha Zeb and Shahzad Amin Sheikh
}

\begin{abstract}
Active noise control (ANC) systems employing adaptive filters suffer from stability issues in the presence of impulsive noise. To overcome this limitation, new methods must be investigated. In this paper, we propose the filtered-x state-space recursive least square (FxSSRLS), an SSRLS-based practical and adaptive algorithm for ANC. Computer simulations are executed to verify the enhanced performance of the FXSSRLS algorithm. Symmetric a-stable (SaS) distributions are used to model impulsive noise. The results show that the proposed FXSSRLS algorithm is more robust in eliminating high-peaked impulses than the recently reported algorithms for ANC applications. Moreover, the suggested solution exhibits better stability and faster convergence, without jeopardizing the performance of the proposed solution in terms of residual noise suppression in the presence of impulses.
\end{abstract}

Keywords: Impulsive noise, Active noise control, Stable distribution, Step size, FxLMS, SSRLS, MNR

\section{Introduction}

Active noise control (ANC) has been extensively used by researchers over the last two decades, due to its superior performance in canceling low-frequency noise as compared to passive methods such as enclosures, barriers, and silencers [1]. Impulsive noise is non-Gaussian in nature, which means that it involves the frequent occurrence of amplitudes that are larger than those found in Gaussian noise. Hence, the information contained within the signal is altered significantly. Power line communication interference, underwater acoustic signals, low-frequency atmospheric noise, noise generated by punching machines, infusion pump sounds in hospitals, and all types of manmade noise can be classified as impulsive noise [2-4].

Figure 1 shows the basic block diagram of a singlechannel feed-forward ANC system using an adaptive algorithm. The system consists of two microphones and a control system. The two microphones are used to obtain reference noise $x(n)$ and residual noise error signal $e(n)$ while the control system is used to generate an antinoise signal $d(n)$. The output $y(n)$ of the adaptive control system drives the cancelation loudspeaker.

\footnotetext{
* Correspondence: alina.mirza78@ceme.nust.edu.pk
Department of Electrical Engineering, College of Electrical and Mechanical
Engineering, National University of Sciences and Technology, Islamabad,

* Correspondence: alina.mirza78@ceme.nust.edu.pk
Department of Electrical Engineering, College of Electrical and Mechanical
Engineering, National University of Sciences and Technology, Islamabad,

* Correspondence: alina.mirza78@ceme.nust.edu.pk
Department of Electrical Engineering, College of Electrical and Mechanical
Engineering, National University of Sciences and Technology, Islamabad, Pakistan
}

The biggest challenges incurred in the ANC of impulsive noise are convergence and stability of the noise reduction algorithms. Due to its simplicity and low computational complexity, the most widely used algorithm for ANC is filtered-x least mean square (FxLMS), which is designed to minimize the variance of error signal [5]. However, since the second-order moment does not exist in case of impulsive noise [6], it cannot be used for impulsive noise reduction. Sun et al. [7] proposed a modification in the FxLMS algorithm which ensures stability of the system. They applied fixed thresholds on the reference signal in order to eliminate the effect of large amplitudes of impulses. Instead of ignoring the samples as in [7], Akhtar and Mitsuhashi [8] improved the Sun algorithm by replacing impulses with new threshold values of error and reference signals to achieve faster convergence along with enhanced stability. The algorithms $[5,7,8]$ are bound to update their threshold parameters during runtime operation which increases computational complexity. To reduce the complexity, another normalized step-size FxLMS (NSS-FxLMS) algorithm is reported in [9], which does not need modified reference or error signal. And in consequence to that, no selection of the threshold parameters are required. Wu et al. in [10] suggested a new technique (FxlogLMS) based on fair M-estimator that minimizes the squared logarithmic transformations of error signal to achieve robustness. However, the algorithm has the 


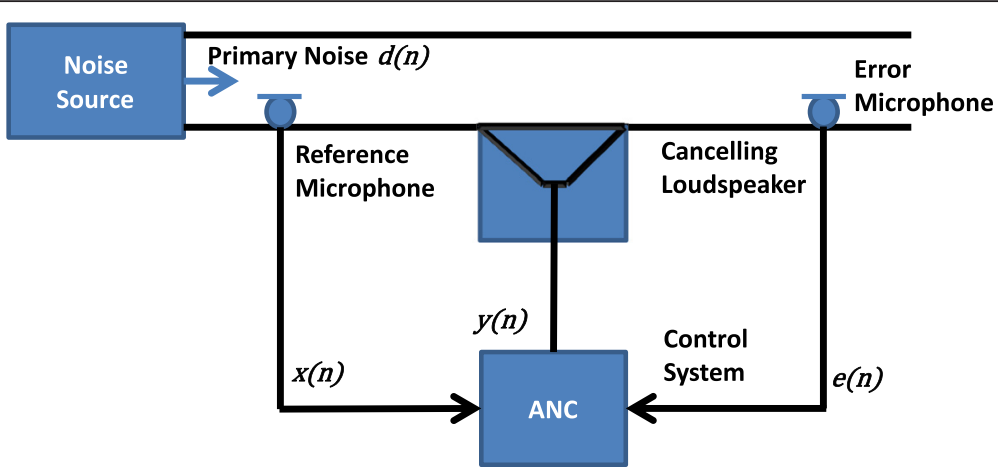

Fig. 1 Basic principle of the feed-forward ANC system

drawback of reaching a dead zone in the process of updating the filter coefficients. Bergamasco et al. [11] provided a solution based on online estimation of the secondary path for ANC applications. A modified Filtered $\mathrm{x}$ Least Mean M-estimator (FxLMM) algorithm [12], established on a two-part skewed triangular M-estimate, is presented to achieve stability in FxLMM when exposed to high-peaked impulses. Data reusing-based normalized step-size FxLMS (DR-NSSFxLMS) algorithm, recommended in [13] for active control of impulsive noise sources, improved the performance but at the expense of increased computational complexity. Similarly, the Filtered $x$ Recursive Least Square (FxRLS) algorithm [14] is used for impulsive noise control, which gives faster convergence but at the cost of increased computational complexity. The Gauss-Siedel algorithm [15] and dichotomous coordinate descent (DCD) algorithm [16] are used for recursive least square adaptive filtering that gives reduced computational complexity. However, the main problem with recursive least square (RLS) family algorithms is the lack of robustness. To enhance the robustness of the RLS algorithm, a modification, i.e., state-space RLS, is presented in $[17,18]$. State-space recursive least square (SSRLS) exhibits excellent tracking performance due to its model-dependent state-space formulation but has not been tested in the ANC domain. Due to the presence of the secondary path $s(z)$ in Fig. 2, SSRLS cannot be used in its existing form. Therefore, in this paper, we have modified the SSRLS algorithm to track the filtered reference noise, making it suitable for ANC applications. The SSRLS algorithm is used in combination with filtered reference input and hence named as the filtered-x SSRLS algorithm.

The rest of the paper is organized as follows: Section 2 presents the proposed algorithm along with its associated schematics. Following this, the simulation results are shown in Section 3. Finally, the conclusions are drawn in Section 4.

\section{Proposed algorithm}

Consider the unforced discrete time system

$$
r[n+1]=\operatorname{Ar}[n]
$$

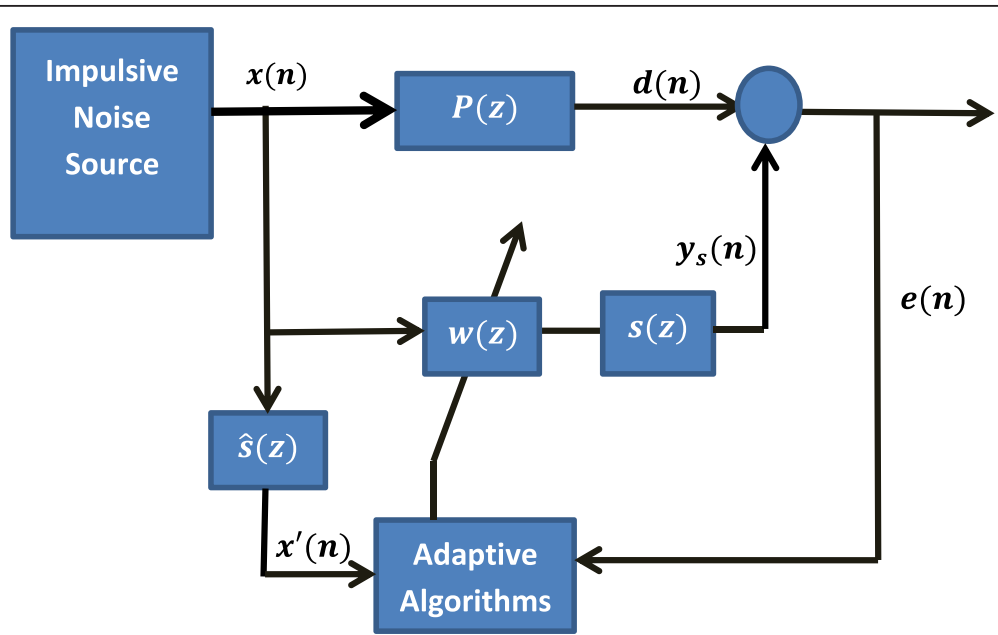

Fig. 2 Block diagram of the ANC system for impulsive noise 


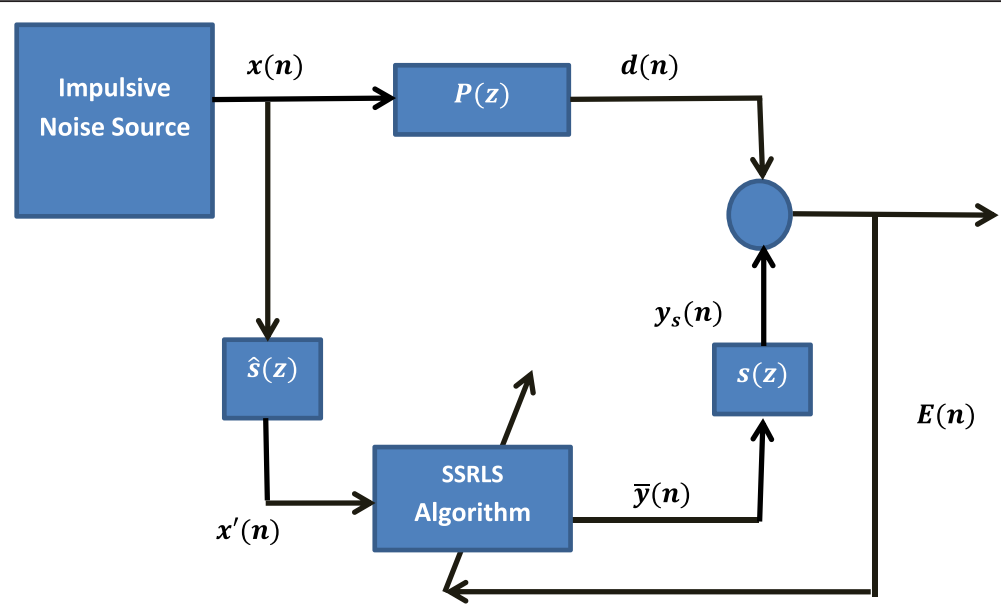

Fig. 3 Block diagram of the proposed algorithm for the ANC system

$$
y[n]=\operatorname{Cr}[n]
$$

where $r$ is the process states and $y$ is the output, while $A$ and $C$ represent the system and the observation matrices, respectively. We assume that the pair $(A, C)$ is L-step observable and $A$ is invertible. The state-space formulation of SSRLS provides the designer with the freedom to choose an appropriate model for the underlying environment. However, the reasons that SSRLS cannot be used in its existing form for active noise control are as under:

1) SSRLS is designed for unforced system, i.e., system without input.

2) ANC applications have a secondary path $s(z)$ following the adaptive filter $w(z)$ as shown in Fig. 2.

3) Impulsive noise state-space model is unknown.

According to the required modifications in SSRLS for the ANC domain, the block diagram of the proposed algorithm is shown in Fig. 3.

In this figure, the reference noise signal vector is $x(n)=[x(n), x(n-1) \ldots \ldots x(n-L+1)]$ ', where $L$ is the length of the reference noise. The desired signal $d(n)$ is calculated as

$d(n)=P(n) * x(n) \quad$ where opeartor $*$ represents the convolution

The filtered reference noise $\boldsymbol{x}^{\prime}(n)=\left[\boldsymbol{x}^{\prime}(n), \boldsymbol{x}^{\prime}(n-1) \ldots .\right.$. $\left.x^{\prime}(n-L+1)\right]^{\prime}$ and the error signal $E(n)$ measured by the error microphone is

$$
\begin{aligned}
& \boldsymbol{x}^{\prime}(n)=\hat{s}(n) * \boldsymbol{x}(n) \\
& E(n)=d(n)-\boldsymbol{y}_{\boldsymbol{s}}(n)
\end{aligned}
$$

The $s(n)$ and $\hat{s}(n)$ are the impulse responses of the secondary path and its estimate, respectively.
Due to the presence of the secondary path following the adaptive filter, phase mismatch occurs between the desired signal and output of the filter as shown in Fig. 3, which consequently degrades the performance of the ANC system. Thus, for incorporating the effect of the secondary path, an identical filter is placed in the reference signal path leading to input of the filter. The modified output of the adaptive filter followed by the secondary path is given by

$$
\boldsymbol{y}_{\boldsymbol{s}}(n)=s(n) * \bar{y}(n)
$$

The filtered reference noise signal $\boldsymbol{x}^{\prime}(n)$ is passed to

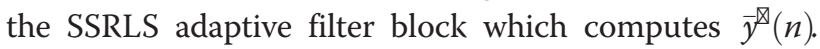
The description of the parameters used for our modified filtered-x state-space recursive least square (FxSSRLS) algorithm are listed in Table 1.

Table 1 Detail of variables

\begin{tabular}{ll}
\hline Variables & Description \\
\hline$P(z)$ & Transfer function of the primary path \\
$S(z)$ & Transfer function of the secondary path \\
$x(n)$ & Reference impulsive noise \\
$S^{\prime}(z)$ & Estimated transfer function of the secondary path \\
$d(n)$ & Desired signal \\
$x^{\prime}(n)$ & Filtered reference noise \\
$\hat{r}[n]$ & Estimated states of the adaptive filter \\
$\bar{r}[n]$ & Predicted states of the adaptive filter \\
$K[n]$ & Gain of the adaptive filter \\
$\varepsilon[n]$ & Prediction error of the adaptive algorithm \\
$\delta$ & Regularization parameter \\
$\lambda$ & Forgetting factor \\
$\bar{y}(n)$ & Predicted output of the adaptive filter \\
$y_{s}(n)$ & Adaptive filter output followed by the secondary path \\
$N$ & Total samples \\
\hline
\end{tabular}


Since the system given in (1) is for forced systems with $x^{\prime}(n)$ being the input, the three special models of SSRLS in $[17,18]$ are modified in Table 2.

After modification of the SSRLS models, the next objective is to select the appropriate model for impulsive noise to get the best match of the underlying environment with the presumed model of SSRLS for achieving enhanced performance of SSRLS. The exact model for impulsive noise cannot be determined because of its random nature. As the higher order models can better approximate the abrupt changes in impulsive noise therefore, we have used an acceleration model in our application. The choice has been validated through extensive simulations. The proposed algorithm is summarized below.

\subsection{Performance analysis and computational complexity}

For adaptive filters, when a new algorithm is developed, it is important to carry out its performance analysis. Although the FxLMS algorithm has been widely used for the implementation of ANC applications, its convergence analysis is still an active area of research [19-21]. The inclusion of the secondary path in FxLMS makes its convergence analysis complex as compared to the standard LMS algorithm. Various attempts on derivation of theoretical convergence analysis for the FxLMS algorithm have been made with different simplified assumptions on inputs being single or multitonal, stationary or purely white, and secondary path being pure delay or moving average model, etc. [22, 23].

\section{Proposed FxSSRLS Algorithm}

\section{Parameters:}

Select A, C, N

Initialize $\delta, \lambda, \mathrm{T}_{\mathrm{s}}, \bar{r}[0]=0, \Phi[0]=\delta \mathrm{I}+\mathrm{C}^{\mathrm{T}} \mathrm{C}, \mathrm{K}[0]=\Phi^{-1}(0) C^{T}, \varepsilon[0]=y[0]-\bar{y}[0]$

\section{Computation:}

While $\{x(n)\}$ available do

$$
\begin{aligned}
& y(n)=p(n) * x(n) \\
& x^{\prime}(n)=\hat{s}(n) * x(n) \\
& \hat{r}[n]=\bar{r}[n]+K[n] \varepsilon[n] \\
& \Phi[n]=\lambda\left(A^{-T} \Phi[n-1]\right) A^{-1}+C^{T} C \\
& K[n]=\Phi^{-1}[n] C^{T} \\
& \bar{r}[n]=A \hat{r}[n] \\
& \bar{y}[n]=C \bar{r}[n] \\
& y_{s}(n)=s(n) * \bar{y}(n) \\
& \varepsilon[n]=y[n]-y_{s}[n]
\end{aligned}
$$

\section{end while}


Table 2 Modified state-space models of SSRLS

\begin{tabular}{lll}
\hline Sinusoidal model & Velocity model & Acceleration model \\
\hline$A=\left[\begin{array}{c}\cos (w T) \sin (w T) \\
-\sin (w T) \cos (w T)\end{array}\right]$ & $A=\left[\begin{array}{ll}1 & T \\
0 & 1\end{array}\right]$ & $A=\left[\begin{array}{ccc}1 & T & \frac{T^{2}}{2} \\
0 & 1 & T \\
0 & 0 & 1\end{array}\right]$ \\
$C=\left[\begin{array}{ll}x^{\prime}(n) & 0\end{array}\right]$ & $C=\left[\begin{array}{ll}x^{\prime}(n) & 0\end{array}\right]$ & $C=\left[\begin{array}{lll}x^{\prime}(n) & 0 & 0\end{array}\right]$
\end{tabular}

The analysis of the SSRLS algorithm for the standard adaptive filter has been presented in $[17,18]$, which may be extended to perform theoretical analysis of the $\mathrm{Fx}$ version of the SSRLS algorithm for the ANC systems. This paper develops a modified SSRLS (FxSSRLS) algorithm for ANC of impulsive sources being modeled as symmetric $\alpha$-stable $(\mathrm{S} \alpha \mathrm{S})$ distributions. For stable distributions, the moments only exist for the order lesser than the characteristic exponent [6], i.e., for impulsive noise, second-order moments do not exist. The lower order moments are more difficult to compute than the second-order moments [24], which makes the theoretical analysis difficult, if not impossible. The nonGaussian signal processing is in general much more complicated in terms of finding statistics than the Gaussian signal processing. This may be the reason that recent work on ANC of impulsive sources (being modeled as stable process) does not include the theoretical analysis, and in fact, the simulations have been used as a major tool to demonstrate the effectiveness of the proposal (see, for example, $[7,8,11])$. The interested reader may also look into the recent works on ANC [25-31]. Though simulations do not prove, they do demonstrate the effectiveness. In this paper, we have also used computer simulations as the evaluation tool and it is observed that the proposed algorithm outperforms the existing algorithms.

Moreover, computational complexity of an algorithm is usually of significant importance particularly in real-time applications. The complexity of individual equations of the proposed FxSSRLS algorithm is given in Table 3, followed by the complexity analysis of other investigated algorithms in Tables 4, 5, 6, and 7.

Here, $L$ represents the total number of states in the FxSSRLS algorithm while in other investigated algorithms, it represents the number of filter coefficients $[17,18] . M$ represents the secondary path, and $N$ represents the data reuse order for the DR-NSSFxLMS algorithm. The computational complexities along with the memory requirements of the investigated algorithms are summarized in Table 8. The memory of the investigated algorithms is calculated using the method given in [32].

Figure 4 shows the plots for the computational complexities of the investigated algorithms. The proposed FxSSRLS algorithm has high computational complexity as compared to the FxLMS and FxRLS algorithm family, which makes it costly for few applications. Nevertheless, in the practical applications where stability and fast convergence is a matter of concern, the implementation of FxSSRLS in the ANC system can be easily handled by the latest DSPs.

\section{Comparison with existing techniques and simulation results}

The ANC system for impulsive noise is implemented using the MATLAB platform. The performance of the proposed algorithm is compared with that of the already reported adaptive algorithms in literature [5, 7-9, 13, 14]. The parameters used in simulating the ANC system are tabularized below.

In our simulation setup, the $\mathrm{S} \alpha \mathrm{S}$ distributions are used to model the statistical parameters of impulsive noise [6]. The analytical form for probability density functions (PDFs) of stable distributions does not exist, so they are normally expressed by their characteristic equations which is actually fourier transform of its PDF.

Table 3 Complexity analysis of the proposed algorithm

\begin{tabular}{|c|c|c|c|c|}
\hline Equations & Operations & * & $+/-$ & $\div$ \\
\hline 1 & $x^{\prime}(n)_{1 \times 1}=s(n)_{1 \times M} * x(n)_{M \times 1}$ & M & $M-1$ & - \\
\hline 2 & $r[n]_{L \times 1}=\bar{r}[n]_{L \times 1}+K[n]_{L \times 1} \varepsilon[n]_{1 \times 1}$ & L & L & - \\
\hline 3 & $\Phi[n]_{L x L}=\lambda\left(A^{-} T_{L x L} \Phi[n-1]_{L x L}\right) A^{-1}{ }_{L x L}+C T_{L x 1} C_{1 \times L}$ & $2 L^{3}+2 L^{2}$ & $2 L^{3}-2 L^{2}$ & 1 \\
\hline 4 & $K[n]_{L x 1}=\Phi^{-1}(n)_{L x L} C T_{L \times 1}$ & $L^{2}$ & $L^{2}-L$ & - \\
\hline 5 & $\bar{r}[n]_{L \times 1}=A_{L \times L} r[n]_{L \times 1}$ & $L^{2}$ & $L^{2}-L$ & - \\
\hline 6 & $\bar{y}[n]_{1 \times 1}=C_{1 \times L} \bar{r}[n]_{L \times 1}$ & $L$ & $L-1$ & - \\
\hline 7 & $y_{s}(n)_{M \times 1}=s(n)_{M \times 1} * \bar{y}(n)_{1 \times 1}$ & M & - & - \\
\hline \multirow[t]{2}{*}{8} & $\varepsilon[n]_{1 \times 1}=y[n]_{1 \times 1}-y_{5}[n]_{1 \times 1}$ & - & 1 & - \\
\hline & Total & $2 L^{3}+4 L^{2}+2 L+2 M$ & $2 L^{3}+M$ & 1 \\
\hline
\end{tabular}


Table 4 Complexity analysis of the FxLMS algorithm

\begin{tabular}{llll}
\hline Equations & Operations & $*$ & $+/-$ \\
\hline 1 & $x^{\prime}(n)_{1 \times 1}=s(n)_{1 \times M} * x(n)_{M \times 1} * x(n)_{M \times 1}$ & $M$ & $M-1$ \\
2 & $y(n)_{1 \times 1}=w T(n)_{1 \times L} * x(n)_{L \times 1}$ & $L$ & $L-1$ \\
3 & $w(n+1)_{L \times 1}=w(n)_{L \times 1}-\mu_{1 \times 1} * e(n)_{1 \times 1} * x^{\prime}(n)_{1 \times L}$ & $L+1$ & $L$ \\
4 & $e(n)_{1 \times 1}=d(n)_{1 \times 1}-y_{s}(n)_{1 \times 1}$ & - & 1 \\
5 & $y_{s}(n)_{1 \times 1}=s(n)_{1 \times M} * y(n)_{M \times 1}$ & $M$ & $M-1$ \\
& Total & $2 L+2 M+1$ & $2 L+2 M-2$ \\
\hline
\end{tabular}

Table 5 Complexity analysis of the NSS-FxLMS algorithm

\begin{tabular}{|c|c|c|c|c|}
\hline Equations & Operations & * & $+/-$ & $\div$ \\
\hline 1 & $x^{\prime}(n)_{1 \times 1}=s(n)_{1 \times M} * x(n)_{M \times 1} * x(n)_{M \times 1}$ & M & $M-1$ & - \\
\hline 2 & $y(n)_{1 \times 1}=w T(n)_{1 \times L} * x(n)_{L x 1}$ & L & $L-1$ & - \\
\hline 3 & $w(n+1)_{\llcorner\times 1}=w(n)_{\llcorner\times 1}-\mu(n)_{1 \times 1} * e(n)_{1 \times 1} * x^{\prime}(n)_{1 \times L}$ & $L+1$ & L & - \\
\hline 4 & $\mu(n)_{1 \times 1}=\frac{\overline{\mu(n)_{1 \times 1}}}{\delta+x^{\top}(n)_{1 \times\llcorner} x^{\prime *(n)_{\lfloor\times 1}+E(n)}}$ & L & $L+1$ & 1 \\
\hline 5 & $e(n)_{1 \times 1}=d(n)_{1 \times 1}-y_{s}(n)_{1 \times 1}$ & - & 1 & - \\
\hline 6 & $y_{s}(n)_{1 \times 1}=s(n)_{1 \times M}{ }^{*} y(n)_{M \times 1}$ & M & $M-1$ & - \\
\hline \multirow[t]{2}{*}{7} & $E(n)_{1 \times 1}=\lambda E(n-1)_{1 \times 1}+(1-\lambda) E^{2}(n)_{1 \times 1}$ & 3 & 2 & \\
\hline & Total & $3 L+2 M+4$ & $3 L+2 M+1$ & 1 \\
\hline
\end{tabular}

Table 6 Complexity analysis of the FxRLS algorithm

\begin{tabular}{|c|c|c|c|c|}
\hline Equations & Operations & * & $+/-$ & $\div$ \\
\hline 1 & $x^{\prime}(n)_{1 \times 1}=s(n)_{1 \times M} * x(n)_{M \times 1} * x(n)_{M \times 1}$ & M & $M-1$ & - \\
\hline 2 & $y(n)_{1 \times 1}=W T(n)_{1 \times L} * x(n)_{L \times 1}$ & L & $L-1$ & - \\
\hline 3 & $w(n+1)_{L \times 1}=w(n)_{L \times 1}+K(n) L_{x} L^{*} e(n)_{1 \times 1}$ & L & L & - \\
\hline 4 & $K(n)_{L \times 1}=\frac{\pi(n)_{L \times 1}}{\lambda+x^{\prime}(n)_{L x \mid} * \pi(n)_{L \times 1}}$ & $2 L$ & L & 1 \\
\hline 5 & $\pi(n) L_{x 1}=p(n-1) L_{x} L^{*} x^{\prime}(n)_{L x 1}$ & $L^{2}$ & $L^{2}-L$ & \\
\hline 6 & $p(n) L_{x} L=\lambda^{-1 *} p(n-1) L_{x} L-\lambda^{-1 *} K(n) L_{x 1}{ }^{*} x^{\prime}(n)_{1 \times L}{ }^{*} p(n-1) L_{x} L$ & $3 L^{2}$ & $2 L^{2}-L$ & 1 \\
\hline 7 & $e(n)_{1 \times 1}=d(n)_{1 \times 1}-y_{s}(n)_{1 \times 1}$ & - & 1 & - \\
\hline \multirow[t]{2}{*}{8} & $y s(n)_{1 \times 1}=s(n)_{1 \times M}{ }^{*} y(n)_{M \times 1}$ & M & $M-1$ & - \\
\hline & Total & $4 L^{2}+4 L+2 M$ & $3 L^{2}+L+2 M-2$ & 2 \\
\hline
\end{tabular}

Table 7 Complexity analysis of the DR-NSSFxLMS algorithm

\begin{tabular}{|c|c|c|c|c|}
\hline Equations & Operations & * & $+/-$ & $\div$ \\
\hline 1 & $x^{\prime}(n)_{1 \times 1}=s(n)_{1 \times M} * x(n)_{M \times 1} * x(n)_{M \times 1}$ & M & $M-1$ & - \\
\hline 2 & $y(n)_{1 \times 1}=w^{T}(n)_{1 \times L} * x(n)_{L x 1}$ & $L$ & $L-1$ & - \\
\hline 3 & $d_{1}(n)_{1 \times 1}=e(n)_{1 \times 1}+s(n)_{1 \times M} * y(n)_{M \times 1}$ & M & M & - \\
\hline 4 & $e_{1}(n)_{1 \times 1}=d_{1}(n)_{1 \times 1}-w_{1}^{T}(n)_{1 \times L} * x^{\prime}(n)_{L \times 1} * x^{\prime}(n)_{L \times 1}$ & L & L & - \\
\hline \multirow[t]{2}{*}{5} & Compute $w_{1}(n+1)_{L \times 1}$ using the DR algorithm in Table 2 from [25] & $N(3 L+4)$ & $N(3 L+2)$ & - \\
\hline & Total & $2 L+2 M+N(3 L+4)$ & $2 L+2 M-2+N(3 L+2)$ & - \\
\hline
\end{tabular}


Table 8 Performance analysis of the investigated algorithms

\begin{tabular}{llll}
\hline Algorithm & Complexity & & \\
\cline { 2 - 3 } & Additions & Multiplications & Memory \\
\hline FxLMS & $2 L+2 M-2$ & $2 L+2 M+1$ & $2(L+M)$ \\
NSS-FxLMS & $3 L+2 M+1$ & $3 L+2 M+4$ & $2(L+M)$ \\
DR-NSSFxLMS & $2 L+2 M-2+N(3 L+2)$ & $2 L+2 M+N(3 L+4)$ & $(N+2) L+3 M$ \\
FxRLS & $3 L^{2}+L+2 M-2$ & $4 L^{2}+4 L+2 M$ & $3 L+2 M$ \\
Proposed FxSSRLS & $2 L^{3}+M$ & $2 L^{3}+4 L^{2}+2 L+2 M$ & $4 L+2 M$ \\
\hline
\end{tabular}

$$
\varphi(t)=e^{-|t|^{\alpha}}
$$

Some PDFs for $\mathrm{S} \alpha \mathrm{S}$ distributions are shown in Fig. 5. The $\mathrm{S} \alpha \mathrm{S}$ distributions have a characteristic exponent parameter $\alpha \quad(0<\alpha<2)$, which controls the spread of the PDF, i.e., a smaller value of $\alpha$ indicates that noise will be more impulsive with a heavier tail.
For the stable distributions, $\alpha$ ranges between 0 and 2. It is characterized as normal distribution for $\alpha=2$, while the distribution is Cauchy for $\alpha=1$. In Fig. 6, impulsive noise generated by the standard $\mathrm{S} \alpha \mathrm{S}$ process with $\alpha=1.65$ is shown while the parameters used for simulating the impulsive noise are mentioned in Table 9.

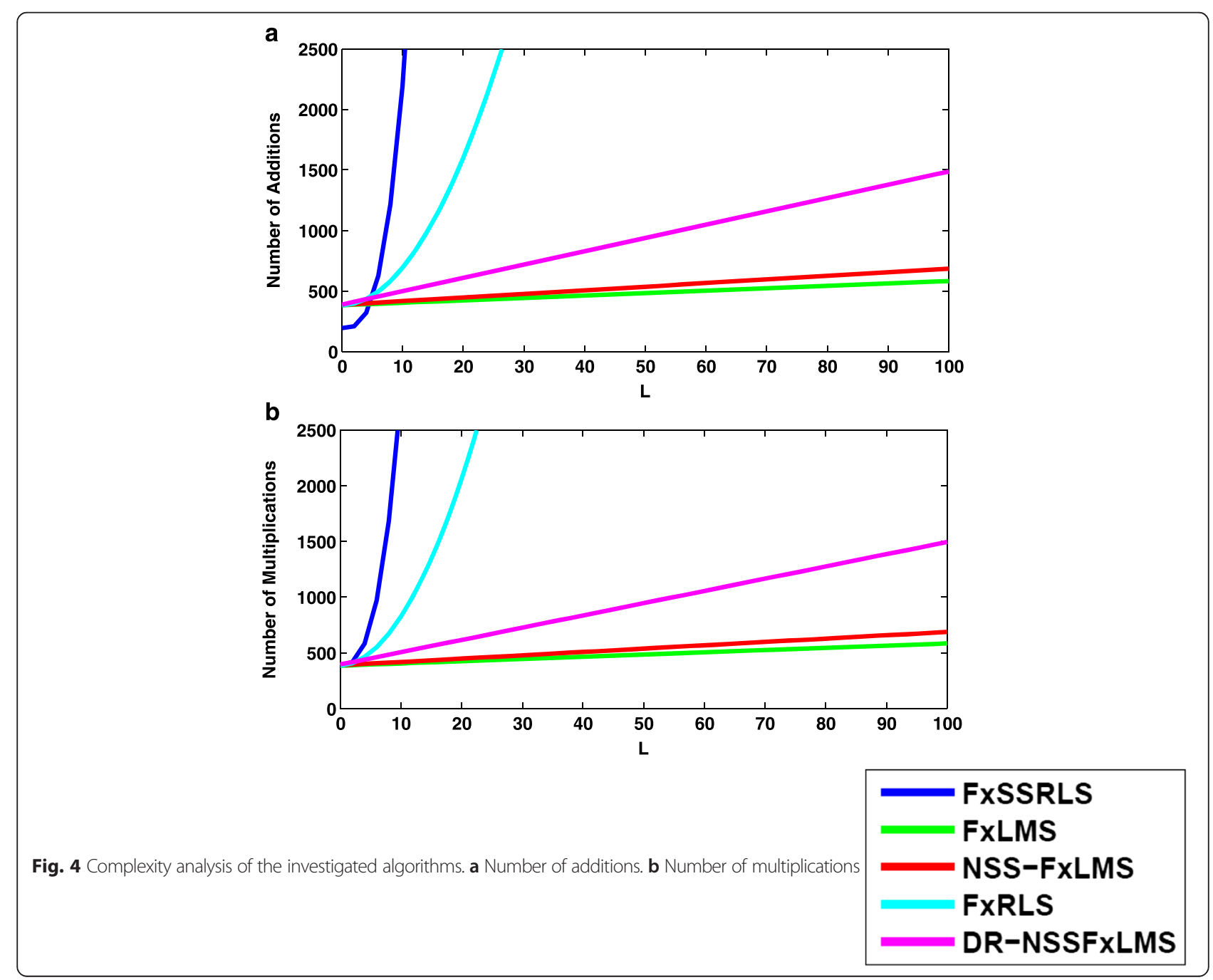




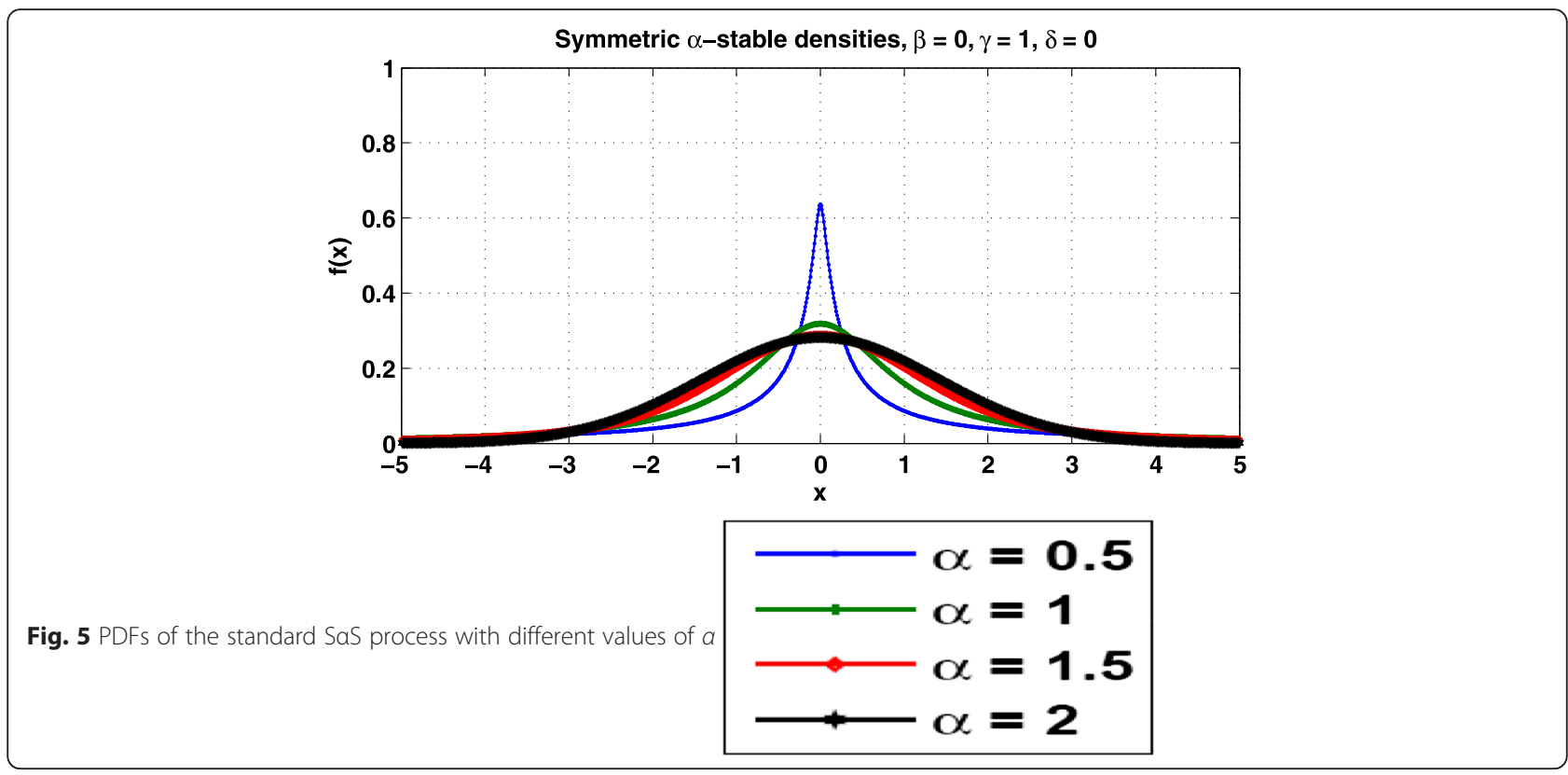

The primary noise $d(n)$ for $\alpha=1.65$ picked by the reference microphone is depicted in Fig. 7.

For the simplicity of our simulations, we have made an assumption that the estimated secondary path model $\hat{s}(z)$ is the same as $s(z)[13,25,30,31]$. The numeric values of the coefficients of the primary and secondary acoustic paths are taken from the data set given in [1]. The frequency response comprising of magnitude and phase of both path filters are depicted in Fig. 8.

The performance metric used in this research for comparison of the studied algorithms is mean noise reduction. It is calculated as

$$
\operatorname{MNR}(n)=E\left\{\frac{A_{e}(n)}{A_{d}(n)}\right\}
$$

$$
\begin{aligned}
& A_{e}(n)=\lambda A_{e}(n-1)+(1-\lambda)|e(n)| \\
& A_{d}(n)=\lambda A_{d}(n-1)+(1-\lambda)|d(n)|
\end{aligned}
$$

where $A_{e}(n)$ and $A_{d}(n)$ are the estimates of the absolute value of the residual error and disturbance signal, respectively.

In this section, we have validated the performance of our proposed algorithm for ANC of impulsive noise. The impulses for the research are generated by the symmetric alpha-stable model by considering $\alpha=1.85, \alpha=1.65$, and $\alpha=1.45$, respectively, which corresponds to a small, mild, and heavy impulsiveness. Extensive simulations are carried out to find the optimum values of controlling the parameters of the discussed algorithms. The detailed simulation results for the step-size parameter of the NSS-FxLMS and DR-NSSFxLMS algorithms for $\alpha=1.65$ are illustrated in

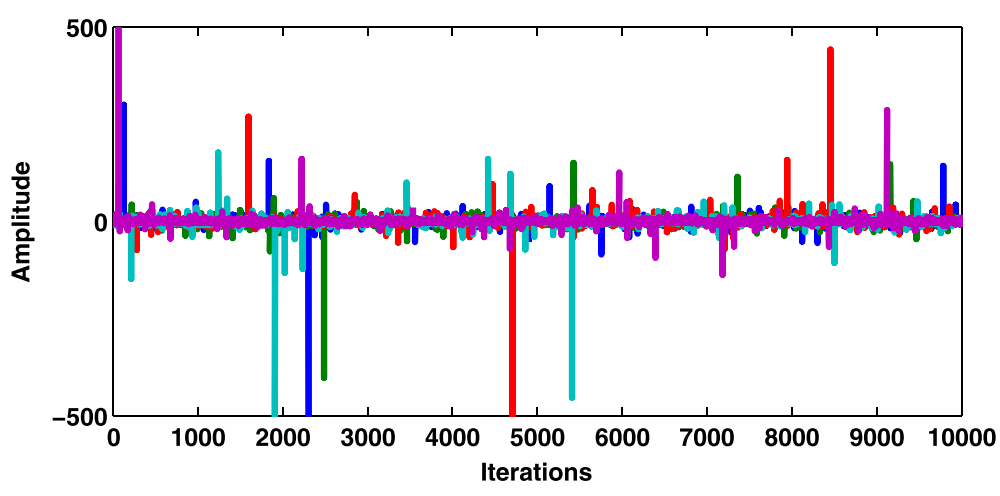

Fig. 6 Impulsive noise generated by the standard SaS process with $a=1.65$ 
Table 9 Parameter set for the proposed technique simulation

\begin{tabular}{|c|c|c|c|c|c|}
\hline \multicolumn{3}{|l|}{ ANC system } & \multicolumn{3}{|l|}{ Impulsive noise } \\
\hline Parameters & Symbols & Values & Parameters & Symbols & Value \\
\hline Primary path tap size & L & 256 & Total samples & $N$ & 10,000 \\
\hline Secondary path tap size & M & 128 & Total realizations & Avg & 1 \\
\hline Adaptive filter tap size & $L_{w}$ & 192 & Characteristic exponent & $a$ & 1.65 \\
\hline DR-NSSFxLMS algorithm step size & $\bar{\mu}$ & $5 e-2$ & Scale parameter & r & 1 \\
\hline NSS-FxLMS algorithm step size & $\bar{\mu}$ & $5 e-2$ & Location parameter & C & 0 \\
\hline RLS forgetting factor & $\lambda$ & 0.99 & Skewness parameter & $\delta$ & 0 \\
\hline SSRLS forgetting factor & $\lambda$ & 1 & & & \\
\hline
\end{tabular}

Fig. 9. It can be observed from Fig. 9 a, b that the optimum step-size value for both algorithms is $5 \mathrm{e}-2$.

Similarly, the effect of the regularization parameter delta $(\delta)$ of the FxRLS algorithm is shown in Fig. 10 for $\alpha=1.65$. The parameter $\delta$ depends on the signal-tonoise ratio (SNR) [5], i.e., the greater the value of the SNR, the smaller the value of delta is selected for better performance of algorithms and vice versa. The optimum value selected for further simulation is 100,000 for $\alpha=1.65$.

Figure 11 depicts the convergence curves of the most widely used adaptive algorithms in the ANC domain for $\alpha=1.65$. The optimum step sizes for the FxLMS, Sun, modified Sun, and Akhtar algorithms used in this simulation are $1 \mathrm{e}-3,5 \mathrm{e}-6,5 \mathrm{e}-5$, and $5 \mathrm{e}-5$, respectively. It can be seen that among the investigated algorithms of the LMS family, the NSS-FxLMS and DR-NSSFxLMS algorithms converge quickly after 1000 iterations and give good noise reduction by achieving the lowest mean noise reduction (MNR) as compared to the other investigated algorithms.
Similarly, the DR-NSSFxLMS algorithm is comparatively less affected by the occurrence of impulsive noise at different iterations and thus exhibits better stability. Therefore, we have selected the NSS-FxLMS and DR-NSSFxLMS algorithms for further comparison with our proposed FxSSRLS algorithm which can be visualized in Fig. 12.

It can be noticed from Fig. 12 that the NSS-FxLMS and DR-NSSFxLMS algorithms give slow convergence as compared to the FxRLS and FxSSRLS algorithms that achieve a steady state value at about 2000 and 500 iterations, respectively. The convergence curves of the FxSSRLS and FxRLS algorithms almost overlap after 3500 iterations. However, when an impulse is encountered at about 800 iterations, the FxRLS algorithm exhibits a sudden increase in MNR while the FxSSRLS algorithm is robust enough to remain unaffected.

Similarly, other simulation cases with $\mathrm{S} \alpha \mathrm{S}$ impulsive noises of $\alpha=1.45$ and 1.85 were conducted to validate the effectiveness of the proposed FxSSRLS algorithm.

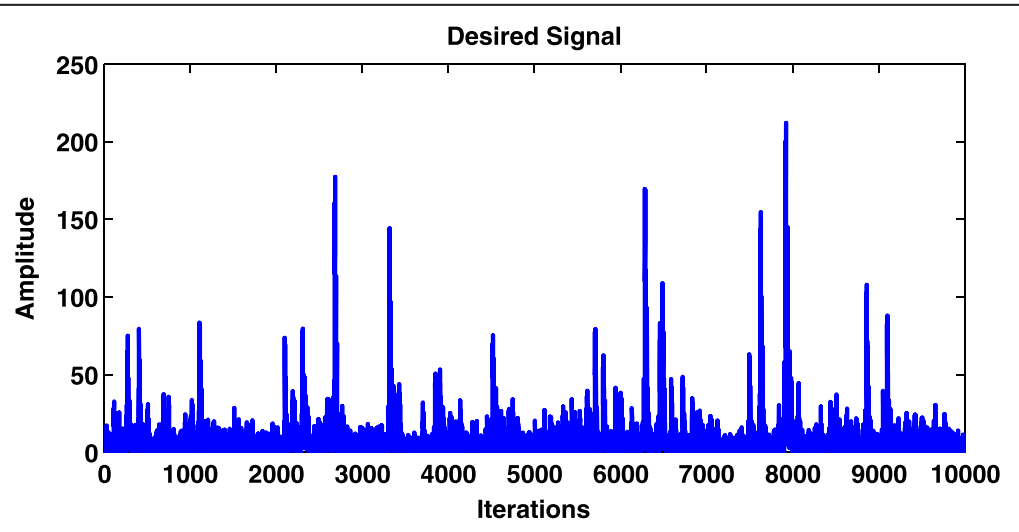

Fig. 7 Primary noise signal 

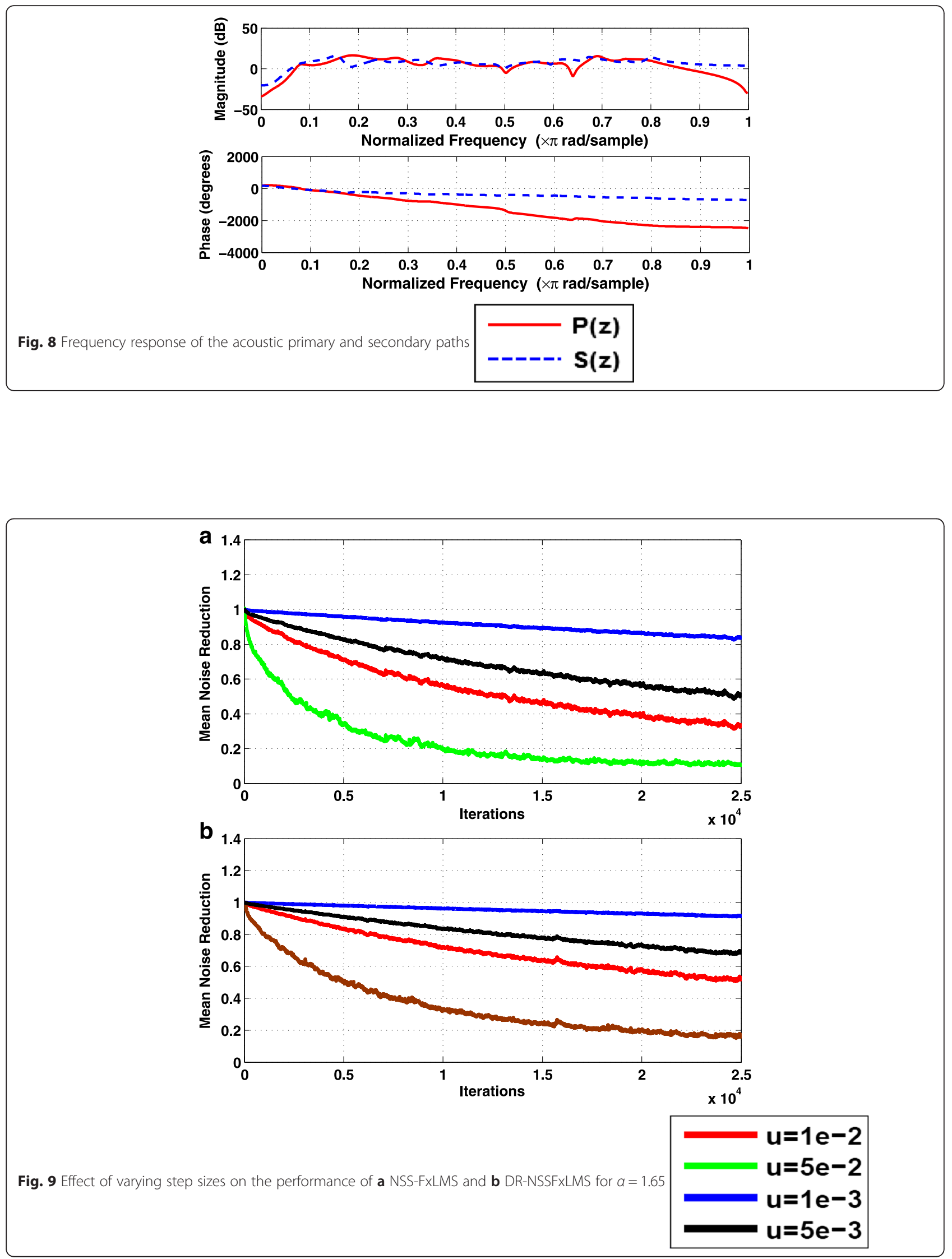

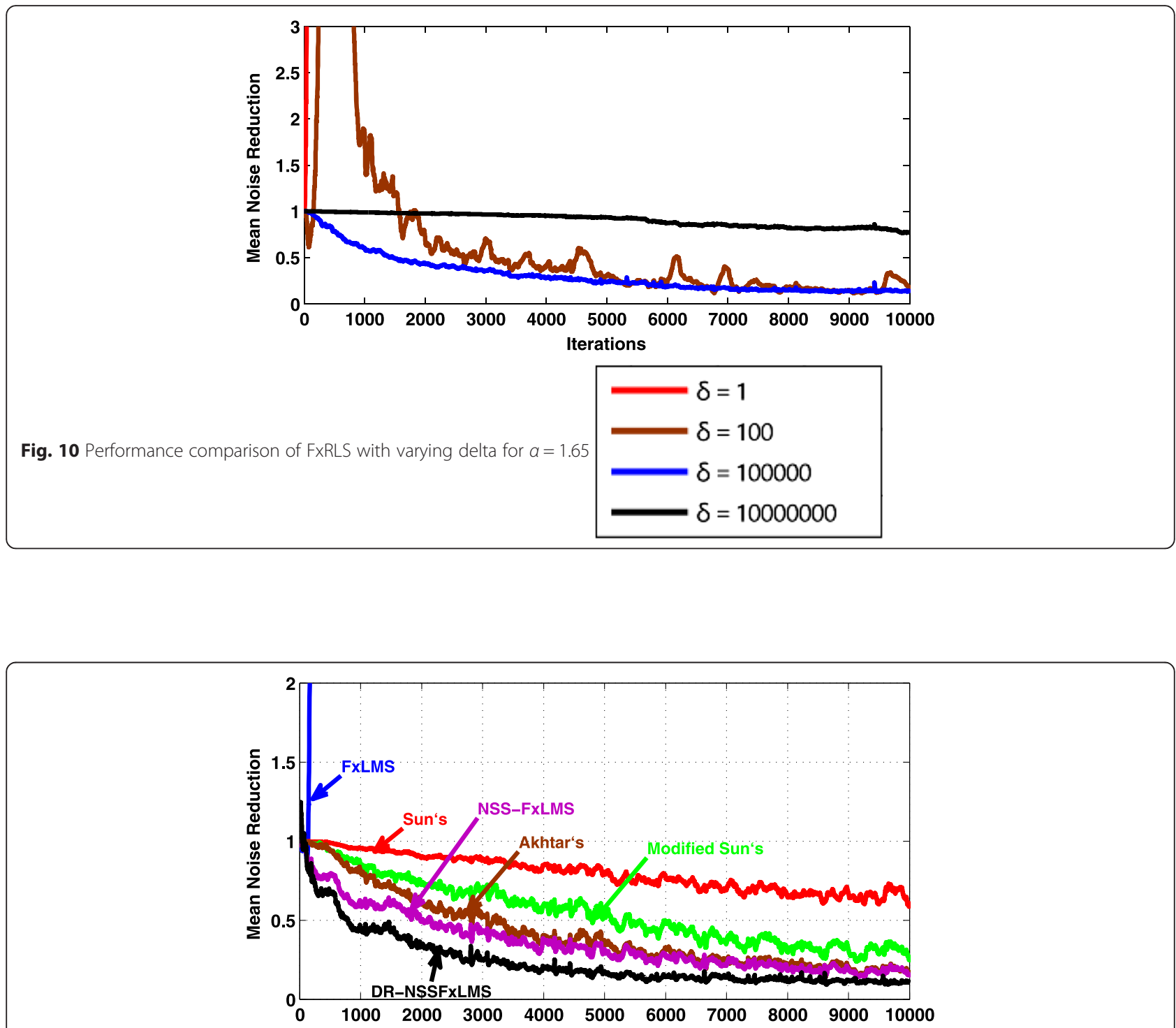

Fig. 11 Convergence curve comparison of various algorithms with $a=1.65$

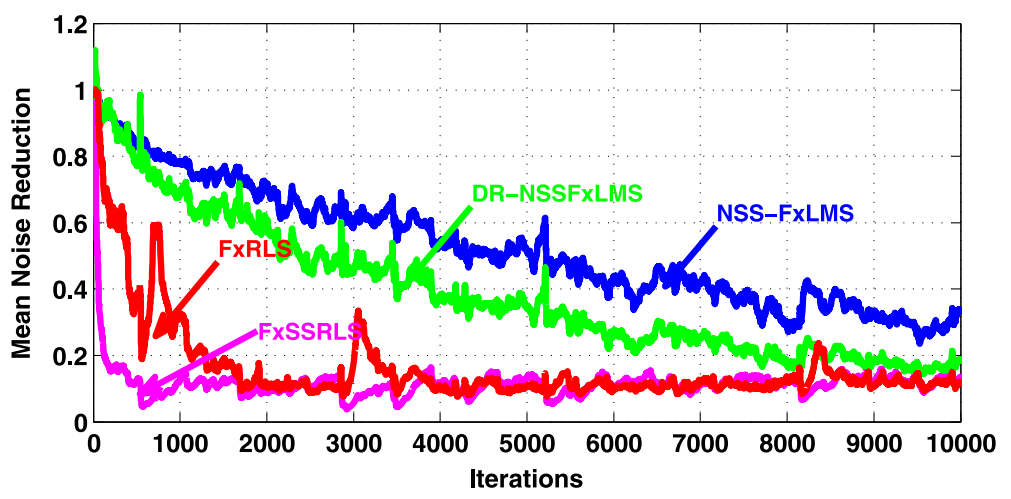

Fig. 12 Comparison of MNR of the FXSSRLS algorithm with various algorithms with $a=1.65$ 


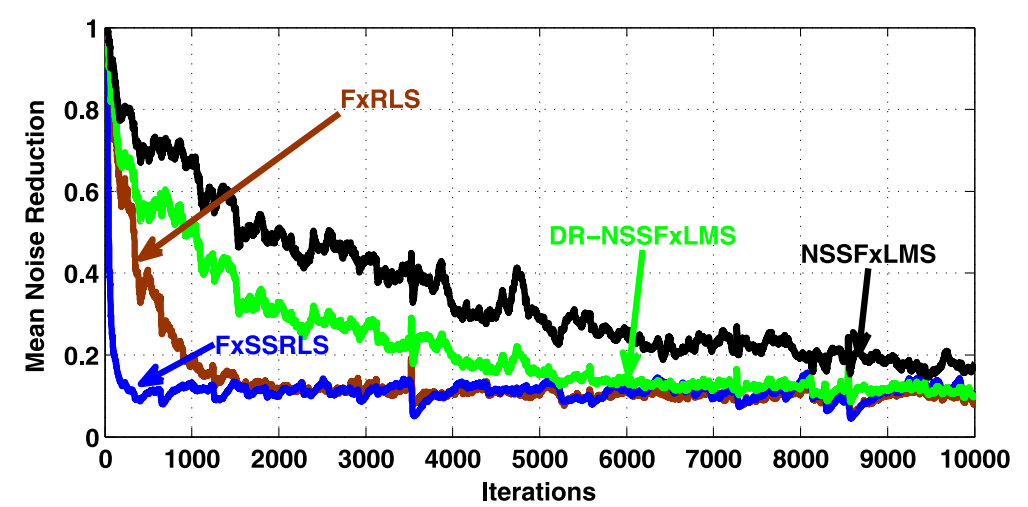

Fig. 13 Comparison of MNR of the FXSSRLS algorithm with various algorithms with $a=1.85$

It is noticed that the proposed FxSSRLS algorithm demonstrates its improved performance for other selected values of impulsive noise over the investigated algorithms. As shown in Figs. 13 and 14, the steady state performance of the FxRLS and proposed FxSSRLS algorithms is better than that of the NSSFxLMS and DR-NSSFxLMS algorithms. Also, the proposed FxSSRLS can yield improved convergence rate and robustness even in the presence of large impulses than that of the FxRLS, NSS-FxLMS, and DR-NSSFxLMS algorithms, thus making our proposed solution an excellent choice for mitigating the influence of impulses in ANC applications.

\section{Conclusions}

In this paper, we have analyzed non-Gaussian impulsive noise in the ANC domain. The adaptive algorithms employed in ANC applications become unstable and lack robustness in the presence of impulsive noise. To overcome this limitation in ANC applications, a new algorithm FxSSRLS has been developed and presented in this paper. Due to the recursive parameters of the proposed adaptive algorithm, the reduction in impulsive noise has been achieved, which has been further enhanced by the state-space formulation of the SSRLS models. To validate this improved performance of the newly suggested solution, extensive numerical simulations have been carried out. The results show that with the use of the presented algorithm for ANC, the large amplitude impulses have been significantly reduced. Moreover, the suggested algorithm for ANC applications outperforms the existing algorithms in terms of mean noise reduction, convergence, and stability. However, this improved performance has been achieved at the cost of slight increase in computational complexity. In applications where stability and fast convergence is a matter of concern, the little price paid in terms of computational complexity can be ignored.

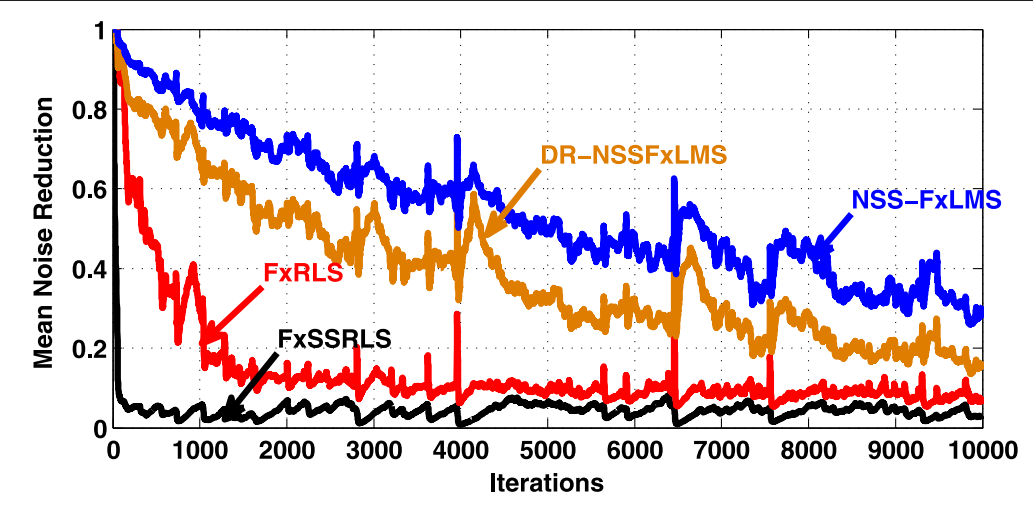

Fig. 14 Comparison of MNR of the FXSSRLS algorithm with various algorithms with $a=1.45$ 


\section{Abbreviations}

ANC: active noise control; DR: data reuse; FxLMS: filtered-x least mean square; FXSSRLS: filtered-x state-space recursive least square; MNR: mean noise reduction; NSS-FXLMS: normalized step-size filtered-x least mean square; PDF: probability density function; RLS: recursive least square; SNR: signal-tonoise ratio; SSRLS: state-space recursive least square; SaS: symmetric a-stable.

\section{Competing interests}

The authors declare that they have no competing interests.

\section{Acknowledgements}

The authors would like to thank their colleague Qasim Umer Khan for improving the content of this paper. The authors would also like to thank the anonymous reviewers for their valuable suggestions and comments.

Received: 3 December 2015 Accepted: 30 March 2016

Published online: 11 April 2016

\section{References}

1. SM Kuo, DR Morgan, Active Noise Control Systems_-Algorithms and DSP Implementation (Wiley, New York, 1996)

2. M Zimmermann, $\mathrm{K}$ Dostert, Analysis and modeling of impulsive noise in broad-band powerline communications. Electromag. Compat. IEEE Trans. 44, 249-258 (2002). doi:10.1109/15.990732

3. L Lui, S Gujjula, P Thanigai, SM Kuo, Still in womb: intrauterine acoustic embedded active noise control for infant incubators. Adv. Acoust Vib (2008). doi:10.1155/2008/495317

4. YI Zhou, YX Yin, QZ Zhang, An optical repetitive control algorithm for periodic impulsive noise attenuation in a non-minimum phase ANC system. Appl. Acoust. 74, 1175-1181 (2013). doi:10.1016/j.apacoust.2013.04.008

5. S Haykin, Adaptive Filter Theory, 4th edn. (Prentice-Hall, Englewood Cliffs, NJ, 2001)

6. CL Nikias, M Shao, Signal Processing with Alpha-Stable Distribution and Applications (Wiley, New York, USA, 1995)

7. X Sun, SM Kuo, M Guang, Adaptive algorithm for active noise control of impulsive noise. J. Sound Vib. 291, 516-522 (2006). doi:10.1016/j.jsv.2005.06.011

8. MT Akhtar, W Mitsuhashi, Improving performance of FXLMS algorithm for active noise control of impulsive noise. J. Sound Vib. 327, 647-656 (2009). doi:10.1016/j.jsv.2009.07.023

9. M.T. Akhtar, W. Mitsuhashi, A modified normalized FxLMS algorithm for active control of impulsive noise. Paper presented at the 18th European signal processing conference, Aalborg, Denmark, 23-27 August 2010

10. $L \mathrm{Wu}, \mathrm{H} H \mathrm{He}, X \mathrm{Qiu}$, An active impulsive noise control algorithm with logarithmic transformation. IEEE Trans. Audio Speech Lang. Process. 19, 1041-1044 (2011)

11. M Bergamasco, FD Rossa, L Piroddi, Active noise control with online estimation of non-Gaussian noise characteristics. J. Sound Vib. 331, 27-40 (2012). doi:10.1016/j.jsv.2011.08.025

12. $\mathrm{P} L i, X Y u$, Active noise cancellation algorithms for impulsive noise. Mech. Syst. Signal Process. 36, 630-635 (2013). doi:10.1016/j.ymssp.2012.10.017

13. MT Akhtar, A Nishihara, Data reusing based filtered reference adaptive algorithms for active control of impulsive noise sources. Appl. Acoust. 92, 18-26 (2015). doi:10.1016/j.apacoust.2015.01.006

14. A Zeb, A Mirza, SA Sheikh, FxRLS algorithm based ANC of impulsive noise. Paper presented at the 7th international conference on modelling, identification and control, Sousse, Tunisia, 18-20 December 2015

15. F Albu, Leading element dichotomous coordinate descent exponential recursive least squares algorithm for multichannel active noise control, Proc. of AAS Acoust. 21-23 November 2012

16. F Albu, C Paleologu, A recursive least square algorithm for active noise control based on the Gauss-Seidel method. Paper presented at the 15th IEEE international conference on electronics, circuits and systems, August 2008

17. MB Malik, State-space recursive least squares: part I. Signal Process. 84, 1709-1718 (2004). doi:10.1016/j.sigpro.2004.05.022

18. MB Malik, State-space recursive least squares: part II. Signal Process. 84, 1709-1718 (2004). doi:10.1016/j.sigpro.2004.05.021

19. LT Ardekani, WH Abdulla, Stochastic modelling and analysis of filtered x-least square adaptive algorithm. IET Signal Process. 7, 486-496 (2013). doi:10.1049/iet-spr.2012.0090

20. G Sun, T Feng, M Li, TC Lim, Convergence analysis of filtered-x least mean squares algorithm for active control of repetitive impact noise. J. Acoust. Soc. Am. 134, 4190-4190 (2013). doi:10.1121/1.4831368
21. G Sun, T Feng, M Li, TC Lim, Convergence analysis of FxLMS-based active noise control for repetitive impulses. Appl. Acoust. 89, 178-187 (2015). doi:10.1016/j.apacoust.2014.09.026

22. LT Ardekani, WH Abdulla, Effects of imperfect secondary path modeling on adaptive active noise control systems. IEEE Trans. Control Syst. Technol. 20, 1252-1262 (2012). doi:10.1109/TCST.2011.2161762

23. LT Ardekani, WH Abdulla, Theoretical convergence analysis of FxLMS algorithm. Signal Process. 90, 3046-3055 (2010). doi:10.1016/j.sigpro.2010.05.009

24. M Shao, CL Nikias, Signal processing with fractional lower order moments: stable processes and their applications. Proc. IEEE 81, 986-1010 (1993). doi:10.1109/5.231338

25. MT Akhtar, Binormalized data-reusing adaptive filtering algorithm for active control of impulsive sources. Digital Signal Process. 49, 56-64 (2015). doi:10.1016/j.dsp.2015.11.002

26. Z Bo, C Sun, Y Xu, S Jiang, A variable momentum factor filtered-x weighted accumulated LMS algorithm for narrow band active noise control systems. Measurement 48, 282-291 (2014). doi:10.1016/j.measurement.2013.11.010

27. SB Behera, DP Das, NK Rout, Nonlinear feedback active noise control for broad band chaotic noise. Appl. Soft Comput. 15, 80-87 (2014). doi:10.1016/j.asoc.2013.10.025

28. L Wu, X Qui, IS Burnett, Y Guo, A recursive least square algorithm for Active control of mixed noise. J. Sound Vib. 339, 1-10 (2015). doi:10.1016/j.jsv.2014.11.002

29. L Wu, X Qui, An M-estimator based algorithm for active impulse like noise control. Appl. Acoust. 74, 407-412 (2013). doi:10.1016/j.apacoust.2012.06.019

30. Y Zhou, Q Zhang, Y Yin, Active control of impulsive noise with symmetric a-stable distribution based on an improved step-size normalized adaptive algorithm. Mech. Syst. Signal Process. 56, 320-339 (2015). doi:10.1016/j.ymssp.2014.10.002

31. G Sun, M Li, TC Lim, A family of threshold based robust adaptive algorithms for active impulsive noise control. Appl. Acoust. 97, 30-36 (2015). doi:10.1016/j.apacoust.2015.04.003

32. SM Kuo, I Panahi, KM Chung, T Horner, M Nadeski, J Chyan, Design of Active Noise Control Systems with the TMS320 family (Texas Instruments, USA, 1996)

\section{Submit your manuscript to a SpringerOpen ${ }^{\circ}$ journal and benefit from:}

- Convenient online submission

- Rigorous peer review

- Immediate publication on acceptance

- Open access: articles freely available online

- High visibility within the field

- Retaining the copyright to your article

Submit your next manuscript at springeropen.com 